\title{
Rehabilitación integral de las personas sentenciadas penalmente para reinsertarlas en la sociedad
}

\author{
Comprehensive rehabilitation of criminally sentenced people to reintegrate them into society
}

Reabilitação abrangente de pessoas condenadas por crime para reintegrá-las à sociedade

Maicol Alejandro Oleas Plaza

maicololeas@hotmail.com

https://orcid.org/0000-0003-0047-9122

Universidad Regional Autónoma de los Andes,

Ambato, Ecuador

\author{
Edison Manuel Pozo Calderon \\ emanuelpozoc@hotmail.com \\ https://orcid.org/0000-0002-0909-693X \\ Universidad Regional Autónoma de los Andes, \\ Ambato, Ecuador
}

Artículo recibido en enero 2021 | Arbitraje en febrero 2021 | Aceptación en marzo 2021 | Publicación en abril 2021

RESUMEN

\section{ABSTRACT}

\section{RESUMO}

La investigación tuvo como objetivo proponer la elaboración de un documento de análisis crítico sobre la falta de presupuestos facticos y jurídicos para lograr la rehabilitación integral de las personas privadas de libertad y lo dispuesto en el artículo 201 de la Constitución de la República del Ecuador. Su metodología fue tipo propositivo, con un enfoque mixto, bajo un diseño documental de campo. Los métodos utilizados fueron; analítico-sistemático, histórico-lógico. Las técnicas usadas fueron la observación y encuesta. La población estuvo compuesta por 13486 abogados inscritos en el foro de abogados de la provincia de Guayas, y la muestra fue de 99 . Se obtuvo como resultado, que no existe un adecuado procedimiento para beneficiarse de la Rehabilitación Social Integral, por lo que los internos, quedan en total desamparo. De esta forma con la propuesta se buscó dar cumplimiento al artículo 201 de la Constitución.

Palabras clave: Rehabilitación Social; derechos y garantías; buen vivir; reclusos; derecho comparado

The objective of the investigation was to determine the need to modify the causes established by article 28 of the Political Constitution of the State for the suspension of the exercise of political rights for crimes against human rights as a legislative mechanism to suppress the idea that the established causes at present they respond to political and ideological objectives of the government of the day. The methodology was descriptive, with a mixed approach, and a documentary design. In addition, the methods used were inductive-deductive, analytical-synthetic, and historical-logical. The techniques and instruments used was the survey (questionnaire). The study population was 190 lawyers and the sample of 89 from the department of Santa Cruz. As a result, the need to carry out the bill was obtained. Since, the protection of the different fundamental rights established in the Constitution is of vital importance for society.

Key words: Social Rehabilitation; rights and guarantees; good living; inmates; comparative law

O objetivo da investigação foi propor a elaboração de um documento de análise crítica sobre a falta de premissas factuais e jurídicas para conseguir a reabilitação integral das pessoas privadas de liberdade e o disposto no artigo 201 da Constituição da República do Equador. A sua metodologia foi do tipo proposicional, com abordagem mista, sob a concepção de documentário de campo. Os métodos usados foram; analítico-sistemático, histórico-lógico. As técnicas utilizadas foram observação e levantamento. A população foi constituída por 13.486 advogados inscritos no fórum de advogados da província de Guayas, e a amostra foi de 99. Como resultado, obteve-se que não existe um procedimento adequado para se beneficiar da Reabilitação Social Integral, portanto, os presidiários ficam em total desamparo. Dessa forma, a proposta buscou atender ao artigo 201 da Constituição.

Palabras-chave: Reabilitação social; direitos e garantias; bom viver; presidiários; direito comparado 


\section{INTRODUCCIÓN}

Se puede decir que la parte de confianza que el estado apuesta al PPL es lo que se denomina, los beneficios carcelarios, los cuales se encuentran señalados en el Código de Ejecución de Penas y Rehabilitación Social, donde se puede encontrar desde rebajas de pena, pre libertad, libertad controlada, y en la Constitución de la Republica de Ecuador en su artículo 51 y todos sus numerales donde se les concede ampliamente derechos que se pueden asimilar.

Sin embargo, en Ecuador no ha existido una verdadera rehabilitación para los internos menos aun que se haya realizado construcción de algún centro para que de esta forma los internos puedan rehabilitarse en su totalidad; $y$ al momento de salir se incorporen a la sociedad como elementos positivos.

No es suficiente que las autoridades penitenciarias se limiten a tratar a los reclusos de manera humana y decente, deben además proporcionar a los internos bajo su custodia, oportunidades de cambiar y desarrollar. Ello requiere considerables aptitudes y un alto nivel de compromiso. Cabe destacar que, la mayoría de las prisiones están llenas de personas procedentes de los márgenes de la sociedad.

Ossorio (2017) define a la Rehabilitación Social de la siguiente manera:

En Derecho Penal, cuando el autor de un delito ha sido condenado a pena que lleve aparejada la inhabilitación, absoluta o especial, puede ser rehabilitado; es decir, restituido al uso y goce de los derechos y capacidades de que fue privado, si cumplida una parte de la condena se ha comportado correctamente. Con carácter más amplio, es la reintegración de la confianza y estima públicas, tras cualquier pena cumplida y cierto plazo adicional, que permita cerciorarse del retorno del condenado a la convivencia social adecuada. No se les concede a los reincidentes, ya que prueban que no la merecían (pág. 831).

En este sentido, los Centros de Rehabilitación deberían ser lugares en las que existan programas integrales de actividades constructivas que ayuden a los internos a mejorar su situación. Como mínimo, la experiencia de la prisión no dejara los reclusos en una situación peor a la que estaban al comenzar su condena, sino que debe ayudarles a mantener y mejorar sus condiciones sanitarias, intelectuales y sociales.

El derecho a la Rehabilitación Social es un sistema establecido en el artículo 201, de la Constitución de la República del Ecuador (2008), su objetivo y finalidad es la rehabilitación integral de las personas sentenciadas penalmente, para reinsertarlas en la sociedad, así como la protección de las personas privadas de libertad y la garantía de sus derechos, es un sistema fundamental del Estado, y hace parte de los mecanismos de participación y control ciudadano. 
A pesar que la norma vigente tiene como objetivo primordial el prometedor funcionamiento de los Centros Penitenciarios con respecto a la rehabilitación y reinserción de las personas privadas de libertad, lo deja en la nada, ya que no se establece un sistema de verificación durante el tiempo de la condena, ni mucho menos después de haber obtenido su libertad; en el que se pueda comprobar y evidenciar que los programas y el tratamiento rehabilitador existentes son los idóneos para tal propósito.

Por lo tanto, la investigación tuvo por objetivo central proponer la elaboración de un documento de análisis crítico sobre la falta de presupuestos facticos y jurídicos para lograr la rehabilitación integral de las personas privadas de libertad y lo dispuesto en el artículo 201 de la Constitución de la República del Ecuador.

La presente investigación propende normar y rectificar la política del Estado, a fin de que la Rehabilitación y Reinserción Social, cumpla con los derechos establecidos en la Constitución de la República del Ecuador, y en Instrumentos Internacionales sobre Derechos Humanos, y de esta manera dar cumplimiento a la Rehabilitación y Reinserción Social.

\section{Referentes teóricos}

\section{Rehabilitación Social}

Según el diccionario, rehabilitación es "un curso de tratamiento, en gran parte terapia física, diseñada para revertir los efectos debilitantes de una herida” (Oxford Pocket, Dictionary, 2017). Esta definición refleja uno de los más comunes pero acotados conceptos de rehabilitación, uno que está centrado en la atención física.

Un segundo significado de rehabilitación, también acotado y predominante en el Derecho, es aquel ligado a ayudar a "una persona que [...] ha sido liberada de prisión [o que aún está en prisión] a readaptarse a la sociedad" (Collins English Dictionary, 2000, pág. 1299).

Mientras que, Constitución de la República de Ecuador (2008), en su Art. 201 dice:

El sistema de rehabilitación social tendrá como finalidad la rehabilitación integral de las personas sentenciadas penalmente para reinsertarlas en la sociedad, así como la protección de las personas privadas de libertad y la garantía de sus derechos. El sistema tendrá como prioridad el desarrollo de las capacidades de las personas sentenciadas penalmente para ejercer sus derechos y cumplir sus responsabilidades al recuperar la libertad (p. 106).

Garantizando de esta manera, como norma suprema y fundamental la consagración del Derecho a la Rehabilitación Social que tendrá como consecuencia inmediata la reinserción social y laboral del reo. 
Con respecto al significado de la Rehabilitacion Social se debe valorar lo siguiente:

- Habilitar de nuevo.

- Autorizar el ejercicio de los derechos suspendidos o quitados.

- Perdonar al indigno, para que pueda heredar, facultad exclusiva del testador.

- Restituir el crédito o buen nombre al que ha sido víctima de un error judicial en causa criminal.

- Restablecer en el antiguo estado.

- Permitir el ejercicio del comercio y la disposición y administración de sus bienes al quebrado.

- Cancelar los antecedentes penales de un delincuente, luego de cumplida la pena y reparados otros efectos del delito. (Ossorio, 2017).

\section{Garantías constitucionales}

Las garantías constitucionales se definen como los medios o instrumentos que la Constitución de la República del Ecuador (2008) pone a disposición de los habitantes para sostener y defender sus derechos frente a las autoridades, individuos o grupos sociales; mientras que las garantías procesales como las instituciones o procedimientos de seguridad creados a favor de las personas, para que dispongan de los medios que hacen efectivo el goce de sus derechos subjetivos.

Tratados Internacionales que se refieren a la Rehabilitación Social

- El derecho a rehabilitación con arreglo al derecho internacional de derechos humanos.

- La rehabilitación con arreglo al derecho de los tratados de derechos humanos de Naciones Unidas.

- La Carta Internacional de Derechos Humanos.

- La Convención de Naciones Unidas contra la Tortura y Otros Tratos o Penas Crueles, Inhumanos o Degradantes.

- Otros tratados de derechos humanos de Naciones Unidas posteriores a la Convención contra la Tortura.

\section{Tratados Internacionales que se refieren Derechos y Garantías}

La jerarquía de los tratados internacionales en el ordenamiento nacional Respecto de la jerarquía de los instrumentos internacionales de derechos humanos y el derecho interno de cada país la doctrina ubica básicamente cuatro tendencias. A saber, la supra constitucionalización; el rango constitucional; la sub constitucionalidad o rango supralegal y la equiparación legal.

Existen abogados en Ecuador que sostienen que la jerarquía de los tratados internacionales en el ordenamiento jurídico está por debajo de la Constitución, pero por sobre la ley. No obstante, otra tendencia afirma que la Constitución vigente asigna a los tratados internacionales, 
específicamente de derechos humanos, un rango constitucional.

El Estado garantizará a todos sus habitantes, sin discriminación alguna, el libre y eficaz ejercicio y el goce de los derechos humanos establecidos en esta Constitución y en las declaraciones, pactos, convenios y más instrumentos internacionales vigentes. Los derechos y garantías determinados en esta Constitución y en los instrumentos internacionales vigentes, serán directa e inmediatamente aplicables por y ante cualquier juez, tribunal o autoridad.

La actual Constitución prevé que anterior a la aprobación de un tratado internacional por parte de la Asamblea Nacional, la Corte Constitucional deberá dictaminar su conformidad o no con la Constitución y que la aprobación de un tratado que exija una reforma constitucional no podrá hacerse sin que antes se haya expedido dicha reforma. En este sentido siempre que se aprueba un tratado internacional este guardará concordancia con la Constitución y por lo mismo tendrá el mismo rango en el ordenamiento jurídico nacional.

\section{Derechos y Garantías Constitucionales}

La proclamación y existencia de los Derechos Humanos ha estado rodeada de una serie de limitaciones para su pleno y eficaz ejercicio, es por eso que en la mayor parte de los países del mundo y en el Ecuador se evidencia el divorcio existente entre los Derechos humanos reconocidos y declarados en la norma Constitucional con su efectiva vigencia en la práctica.

\section{Derecho al Buen Vivir}

Calderón (2017) se refiere al Buen vivir de la siguiente manera: el Estado es constitucional con derechos y justicia social, democrático, soberano, independiente, su soberanía radica en el pueblo, los recursos naturales no renovables pertenecen a su patrimonio inalienable, irrenunciable e imprescriptible.

Las personas, comunidades y pueblos gozarán de los derechos garantizados en la Constitución y en los instrumentos internacionales y se podrán ejercer y exigir ante las autoridades competentes para su cumplimiento.

A su vez, la Constitución de Ecuador (2008), en su artículo 33 expresa que, entre los derechos del Buen Vivir constitucionalmente se tiene; agua y alimentación, ambiente sano, comunicación e información, cultura y ciencia, educación, vivienda, salud y trabajo y seguridad social. Para poder disfrutar de todos los derechos del Buen Vivir es necesario que las personas tengan trabajo, que es fuente de realización personal y base de la economía y producción de una nación.

El Estado garantizará a las personas trabajadoras el pleno respeto a su dignidad, una vida decorosa, remuneraciones y retribuciones justas y el desempeño de un trabajo saludable y libremente escogido o aceptado. 
La relación que tiene la rehabilitación social de las personas privadas de libertad con el derecho del buen vivir es la atención de sus necesidades educativas, laborales, productivas, culturales, alimenticias y recreativas, como lo determina la misma Constitución en su art. 51.

\section{Rehabilitación y Reinserción Social en el Ecuador}

El sistema penitenciario hace 8 años tenía un panorama poco alentador, donde se evidenciaba la falta de capacidad por parte de las instituciones para sanear la crisis del sistema a nivel nacional. Bajo este escenario, se permite hacer un balance para crear nuevas políticas para la transformación del sistema penitenciario con estricto apego a los derechos constitucionales, crear y aplicar mecanismos que garanticen la seguridad de las personas que cumplen su internamiento.

Con el nuevo modelo de gestión del sistema penitenciario lo que se está constituyendo es lograr ejecutar un proceso de "tratamiento individualizado" a las personas privadas de libertad, para su "rehabilitación e inclusión económica y social", y así cumplir con los objetivos del sistema.

El modelo de gestión penitenciaria se basa en la política del plan nacional para el buen vivir que tiene por objetivo impulsar un sistema de rehabilitación social que posibilite el ejercicio de derechos y responsabilidades de las personas privadas de libertad.

\section{Rehabilitación y Reinserción Social en el Derecho Comparado}

\section{República Dominicana}

El Modelo de Gestión Penitenciaria de República Dominicana muestra evidencias de un cambio positivo, basado en el respeto a los derechos y dignidad de los privados de libertad, un país con un bajo índice de reincidencia del $2.7 \%$, sin duda este modelo sustentado en los principios básicos para el tratamiento de los reclusos adoptados y proclamados por la Asamblea General de las Naciones Unidas en su resolución 45/111, del 14 de diciembre de 1990, ha dado los primeros pasos para la reforma y modernización del Sistema Penitenciario Dominicano, dotando de gran profesionalidad al Cuerpo de Agentes de Vigilancia y Tratamiento Penitenciario, personal Técnico y Administrativo, sin embargo es un modelo que normaliza los establecimientos penitenciarios de forma progresiva donde el único fin es el de elevar los niveles de seguridad pública de la sociedad dominicana.

\section{Noruega}

El Sistema Penitenciario de Noruega conocido por su sistema penitenciario verdaderamente 
humano, donde los internos o personas privados de su libertad cumplen su condena en una islaprisión Bastoy en Noruega donde existen alrededor de 215 internos cumpliendo una condena y su nivel de reincidencia es de un $16 \%$, un país cuyo objetivo no es la de castigar sino el cambiar o el de crear una situación diferente en los prisioneros donde ellos sean capaces de abrirse y de empezar una nueva vida.

La idea de esta prisión es que funcione como un pequeño pueblo autosustentable donde los internos reciben 59 coronas noruegas por día, esto equivale a \$10 dólares. Su filosofía solo puede resumirse en una sola frase: "Si les tratas como hombres, se comportarán como hombres".

MÉTODO

La metodología de la investigación fue de tipo propositivo, ya que se intentó actuar sobre la realidad concreta para mejorarla y/o solucionarla. Bajo un enfoque mixto, cuali-cuantitativo, porque se trató de una investigación de carácter social en el que intervienen la subjetividad del investigador.

El diseño de la investigación fue documental de campo. La primera porque fueron estudios predominantemente teóricos. Su propósito fue desarrollar la teoría con base en datos empíricos obtenidos en la propia investigación, más que en estudios previos. Y el segundo, porque permitió el acercamiento al problema a investigar y la relación objetiva entre las variables utilizando métodos e instrumentos apropiados.

A su vez, los métodos utilizados fueron analítico-sistemático, histórico-lógico e inductivodeductivo.

Las técnicas e instrumentos utilizados fueron la observación científica para su medición, se utilizó el análisis documental y la validación por vía de expertos, que en este caso fueron profesionales del Derecho. La encuesta, por medio del instrumento el cuestionario, fue configurado con preguntas tendientes a demostrar la existencia del problema y dar sustento a la propuesta.

La realización de la investigación se llevó a cabo en el cantón Guayaquil, provincia de El Guayas, por lo que se contó con una población de 13486 abogados inscritos en el foro de abogados de la provincia antes mencionada, mientras que la muestra seleccionada estuvo conformada por 99 abogados.

RESULTADOS

Los resultados obtenidos posterior a la aplicación del instrumento efectuadas a los profesionales inscritos en el foro de abogados de la provincia Guayas fueron: 
En la Tabla 1 se muestra que, el $94 \%$ de los encuestados conocen que la Rehabilitación Social Integral se encuentra establecida en la Constitución de la República del Ecuador, mientras que un $6 \%$ manifiesta lo contrario.

Tabla 1. La Rehabilitación Social Integral se encuentra establecida en la Constitución de la República del Ecuador.

\begin{tabular}{ccc}
\hline ALTERNATIVAS & FRECUENCIA & PORCENTAJE \\
\hline Si & 90 & $94 \%$ \\
No & 9 & $6 \%$ \\
Total & $\mathbf{9 9}$ & $\mathbf{1 0 0 \%}$ \\
\hline
\end{tabular}

En este sentido, se evidenció que existe conocimiento del Derecho a la Rehabilitación Social Integral, establecido en la Constitución de la República del Ecuador (2008). No existe un adecuado procedimiento para beneficiarse de la Rehabilitación Social Integral, por lo que los internos, quedan en total desamparo, siendo necesario buscar una solución al vacío legal y procesal existente.

En la Tabla 2, se expresa que, el $95 \%$ de los encuestados consideran que la normativa que actualmente regula el derecho a la rehabilitación social integral vulnera derechos constitucionales, mientras que el $5 \%$ manifiesta lo contrario.

Tabla 2. La normativa que actualmente regula el Derecho a la Rehabilitación social Integral vulnera derechos y garantías constitucionales.

\begin{tabular}{ccc}
\hline ALTERNATIVAS & FRECUENCIA & PORCENTAJE \\
\hline Si & 93 & $95 \%$ \\
No & 6 & $5 \%$ \\
Total & $\mathbf{9 9}$ & $\mathbf{1 0 0 \%}$ \\
\hline
\end{tabular}

Se demostró la importante que es la elaboración de una resolución administrativa por parte del Ministerio de Justicia, Derechos Humanos y Cultos, estableciendo un adecuado procedimiento para la Rehabilitación Social Integral, con la finalidad de garantizar los derechos y garantías constitucionales.

Se muestra en la Tabla 3 que, el $98 \%$ de los encuestados consideran que la normativa que regula la Rehabilitación social vulnera el artículo 201 de la Constitución de la República del Ecuador, mientras que el $2 \%$ de los encuestados consideran que no es así. 
Tabla 3. La normativa que regula la Rehabilitación social vulnera el artículo 201 de la Constitución de la República del Ecuador.

\begin{tabular}{ccc}
\hline ALTERNATIVAS & FRECUENCIA & PORCENTAJE \\
\hline Si & 96 & $98 \%$ \\
No & 3 & $2 \%$ \\
Total & $\mathbf{9 7}$ & $\mathbf{1 0 0 \%}$ \\
\hline
\end{tabular}

Se evidenció que la normativa que garantiza el derecho de petición vulnera el derecho a recibir servicios públicos de calidad.

En la Tabla 4 se devela que el $64 \%$ de los encuestados considera que existe corrupción, mientras que el $23 \%$ considera que existen vacíos legales y el $13 \%$ considera que falta presupuesto para lograr la rehabilitación social integral.

Tabla 4. Los motivos por cual la normativa que garantiza la Reinserción Social y Rehabilitación Social Integral vulnera derechos Constitucionales

\begin{tabular}{ccc}
\hline ALTERNATIVAS & FRECUENCIA & PORCENTAJE \\
\hline Corrupción & 63 & $64 \%$ \\
Vacíos legales & 23 & $23 \%$ \\
Falta de presupuesto & 13 & $13 \%$ \\
Total & $\mathbf{9 9}$ & $\mathbf{1 0 0 \%}$ \\
\hline
\end{tabular}

Con los resultados obtenidos se evidenció que existe mucha corrupción para acceder a la Rehabilitación Social Integral, en cambio la minoría se pronuncia que no existen vacíos legales y falta de presupuesto, que obviamente inciden en la Rehabilitación Social.

Se muestra en la Tabla 5 que, el $69 \%$ de los encuestados considera que faltan leyes y resoluciones administrativas para acceder a la Rehabilitación Social Integral, mientras que el $21 \%$ considera que falta infraestructura y el $10 \%$ considera que falta tecnología.

Tabla 5. Aspecto que debe mejorarse para lograr la Rehabilitación Social Integral.

\begin{tabular}{ccc}
\hline ALTERNATIVAS & FRECUENCIA & PORCENTAJE \\
\hline Leyes o resoluciones administrativas & 68 & $69 \%$ \\
Infraestructura & 21 & $21 \%$ \\
Tecnología & 10 & $10 \%$ \\
Total & $\mathbf{9 9}$ & $\mathbf{1 0 0 \%}$ \\
\hline
\end{tabular}

Se demostró que existen muchas formalidades para acceder al Derecho Constitucional de Petición, vulnerando el derecho a recibir servicios públicos de calidad. 
Según la Tabla 6, se determina que el $99 \%$ de los encuestados consideran que el derecho a la rehabilitación social integral debe estar debidamente normado, mientras que el $1 \%$ de los encuestados consideran que no es necesario.

Tabla 6. El derecho a la Rehabilitación social integral se encuentre debidamente normado (indispensable)

\begin{tabular}{ccc}
\hline ALTERNATIVAS & FRECUENCIA & PORCENTAJE \\
\hline $\mathrm{Si}$ & 98 & $99 \%$ \\
No & 1 & $\mathbf{1} \%$ \\
Total & $\mathbf{9 9}$ & $\mathbf{1 0 0 \%}$ \\
\hline
\end{tabular}

Se evidenció que es necesario que el derecho a la rehabilitación social integral esté debidamente normado.

Según la Tabla 7, se determina que el $99 \%$ de los encuestados consideran que con la elaboración de un documento de análisis crítico que muestra cómo la falta de presupuestos fácticos y jurídicos afecta a la rehabilitación integral de las personas privadas de libertad y lo dispuesto en el artículo 201 de la Constitución de la República del Ecuador, se garantizará la Rehabilitación Social Integral, mientras que el $1 \%$ de los encuestados consideran que no es necesario.

Tabla 7. La elaboración de un documento de análisis crítico que muestra como la falta de presupuestos facticos y jurídicos afecta a la rehabilitación integral de las personas privadas de libertad y lo dispuesto en el artículo 201 de la Constitución de la República del Ecuador, garantiza la Rehabilitación Social Integral.

\begin{tabular}{ccc}
\hline ALTERNATIVAS & FRECUENCIA & PORCENTAJE \\
\hline $\mathrm{Si}$ & 98 & $99 \%$ \\
No & 1 & $\mathbf{1} \%$ \\
Total & $\mathbf{9 9}$ & $\mathbf{1 0 0 \%}$ \\
\hline
\end{tabular}

En este sentido, se demostró la necesidad de la elaboración de un acuerdo o resolución administrativa del Ministerio de Justicia, Derechos Humanos y Cultos, ya que garantizará la rehabilitación integral de las personas privadas de libertad y lo dispuesto en el artículo 201 de la Constitución de la República del Ecuador. 
Propuesta resolución administrativa del Ministerio de Justicia, Derechos Humanos y Cultos, se garantizará la rehabilitación integral de las personas privadas de libertad y lo dispuesto en el artículo 201 de la Constitución de la República del Ecuador.

\section{Objetivo}

Elaboración de un acuerdo o resolución administrativa del Ministerio de Justicia, Derechos Humanos y Cultos, se garantizará la rehabilitación integral de las personas privadas de libertad y lo dispuesto en el artículo 201 de la Constitución de la República del Ecuador.

\section{Justificación}

A través de la elaboración de un acuerdo o resolución administrativa del Ministerio de Justicia, Derechos Humanos y Cultos, se garantizará la rehabilitación integral de las personas privadas de libertad y lo dispuesto en el artículo 201 de la Constitución de la República del Ecuador.

Si bien la Constitución de la República del Ecuador (2008) se refiere al derecho a la rehabilitación social integral no existe un marco legal adecuado que la respalde y que establezca procedimientos claros rápidos y sencillos para acceder a esta garantía constitucional. Esto produce que se vulneren múltiples derechos constitucionales establecidos en el artículo 51 de la Constitución en su numerales 5, 6, como el buen vivir, entre otros consagrados en la Constitución.

Es por esta razón que es sumamente necesario establecer un marco legal y un procedimiento rápido moderno y sencillo para que los centros de rehabilitación cumplan sus objetivos y fines establecidos en la Constitución de la República del Ecuador del 2008. 
PROPUESTA:

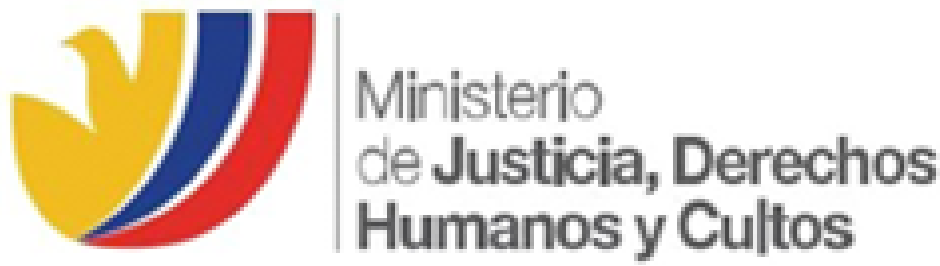

Acuerda:

Expedir el Acuerdo Ministerial Nro. MJDHC-MJDHC-2018-0001-A, de 24 de enero de 2018.

Articulo 1.- Que el estado adopte como politica pública el trabajo para los privados de libertad en los centros penitenciarios del pais siendo prioridad su contratación, en el desarrollo de mano factura, arte, agricultura,

Articulo 2,- Que el privado de liberta una vez que el estado o la empresa privada contrate con el centro de privación de libertad sus beneficios laborales sean cumplidos como la seguridad laboral y todos sus beneficios de ley.

Articulo 3.- Que la remuneración del privado de libertad comience con un salario bísico unificado y no supere los 5 salarios básicos unificados a la fecha de ru condena.

Articulo 4.- Que la remuneración obtenida por el privado de libertad se dividirá de la siguiente manera el $30 \%$ será depositado en su fondo de condena que será retirado unicamente en el momento que recobre su libertad, el $20 \%$ seri destinado para la reparación integral de la victima, si el juzzador en su resolución destina un monto en el cual el privado de libertad antes o durante de su condena se hubiera cancelado en su totalidad, ese $20 \%$ pasara a su fondo de condena, el $50 \%$ sera destinado para el núcleo familiar que haya conformado el privado de libertad.

Artículo 5.- Trabajo en libertad será tomado en cuenta como requisito dentro de los beneficios carcelarios, siendo el estado por medio de la empresa pública o privada que asegure que tena un trabajo digno, este articulado se someteri a la aplicación del tiempo que los beneficios carcelarios rigen.

Articulo 6- Los centros de privación de libertad deberán de estar a cargo de sociólogos que tendrin asesoria obligatoria de abogados, economistas y trabajadores sociales, que serán responsables administrativos del proyecto de trabajo entre las empresas públicas o privadas.

Articulo 7,- El centro de privación de libertad debera tener un minimo del $60 \%$ de sus privados de libertad en actividades desarrolladas al trabajo para las empresas públicas o privadas del estado, el personal escogido para el trabajo debe ser capacitado por expertos en cada rama, con un tiempo mino de 90 antes de entrar en funciones.

Figura 1. Propuesta. 
Articulo 8.- Los privados de libertad podrin asociarse a si de esta manera poder participar en concursos para contrataciones en el SERCOP y poder brindar un trabajo de calidad y calidez.

Articulo 9.- El director del centro de privación de libertad será el responsable directo de la participación de las asociaciones en referente a coutrataciones con el ertado.

Articulo 10-- los centros de privación de libertad una vez adjudicados contratos o los privados de libertad una vez contratados se someterin a los reglamentos intemos que regulen dichas funciones para poder brindar un servicio de calidad y calidez.

\section{DISPOSICIONES GENERALES}

Primera. - Toda disposición contenida en el Acuerdo Ministerial No. 0000 , de 24 de abril de 2017, publicado en el Registro Oficial No. 609, de 16 de octubre de 2015 que no ba sido reformada por el preseate Acuerdo, mantiene su vigencia por lo que zoza de igual valor $y$ efecto legal.

Segunda. - La Dirección de Gestión de Cambio de Cultura Organizacional tendra 30 dias a partir de la suscripción del preseate Acuerdo para entablecer el proceso y mecamismos de autoevaluación correspondiente.

Tercera. - De la ejecución, cumplimiento y seguimiento del presente Acuerdo eacarguese a todas las autoridades constantes en el articulo 3 del Acuerdo Mininterial Nro. 0922.

\section{DISPOSICIÓN FINAL}

El presente Acuerdo, entrará en vigencia a partir de la fecha de su suscripción, sin perjuicio de su publicación en el Registro Oficial. Dado en Quito, D.M. , a los 24 dia(s) del mes de enero de dos mil diez y ocho.

Documento flumedo electónicamente

SRA. DRA. ROSANA ALVARADO CARRION

MINISTRA DE JUSTICLA, DERECHOS HUMANOS Y CULTOS

Figura 2. Propuesta. 


\section{CONCLUSIONES}

Si bien la Constitución de la República del Ecuador establece el Derecho a la Rehabilitación Social Integral, el legislador no se ha preocupado de adecuar la normativa constitucional con la normativa legal o administrativa para garantizar el ejercicio pleno del Derecho a la rehabilitación Social Integral.

En este orden de ideas, el Derecho a la Rehabilitación Social Integral debe cumplir su función y ser una herramienta que, en forma ágil, rápida y sencilla, permita la vinculación de los internos con el resto de la ciudadanía y la administración pública o privada.

Por lo tanto, mediante el acuerdo o resolución administrativa del Ministerio de Justicia, Derechos Humanos y Cultos, se garantizará la rehabilitación integral de las personas privadas de libertad y lo dispuesto en el artículo 201 de la Constitución de la República del Ecuador.

\section{REFERENCIAS}

Asamblea Nacional Constituyente reunida en Montecristi. (2008). Constitución de la Republica del Ecuador. Montecristi-Manabí: Registro Oficial

Calderón, A.(2017) El buen vivir. http://www. eltelegrafo.com.ec/noticias/cartas-aldirector/1/derechos-del-buen- vivir. Obtenido de http://www.eltelegrafo.com.ec/noticias/ cartas-al- director/1/derechos-del-buenvivir: http://www.eltelegrafo.com.ec/noticias/ cartas-al-director/1/derechos-del-buen- vivir
Collins English Dictionary. (2000). Collins English Dictionary. Londres-UK: Harper Collins Publisher

Ossorio, M. (2017). Diccionario de ciencias jurídicas políticas y sociales. Guatemala-Guatemala: Datascan S.A

Oxford Pocket, Dictionary. (2017). Oxford Pocket Dictionary. Obtenido de http://www. encyclopedia.com/doc/10999-rehab.html 